\title{
Effect of Postural Changes on Cardiorespiratory Coordination in Humans
}

\author{
Muammar M. Kabir, Derek \\ Abbott \\ School of Electrical and Electronic \\ Engineering \\ University of Adelaide \\ Adelaide, Australia \\ e-mail: \\ muammar.kabir@adelaide.edu.au, \\ dabbott@eleceng.adelaide.edu.au
}

\author{
David A. Saint \\ School of Medical Sciences \\ University of Adelaide \\ Adelaide, Australia \\ e-mail: \\ david.saint@adelaide.edu.au
}

\author{
Mathias Baumert \\ School of Medicine, School of \\ Paediatrics and Reproductive \\ Health \\ University of Adelaide \\ Adelaide, Australia \\ e-mail: \\ mathias.baumert@adelaide.edu.au
}

\begin{abstract}
In this study we assessed the effect of body postural changes from the supine (Sup) to the upright ( $U p)$ position on the magnitude and phase of respiratory sinus arrhythmia (RSA) and the phase-locking between the heart rhythm and respiration. In eight healthy human subjects, electrocardiograms (ECG) and respiratory signals were recorded. The magnitude and phase of RSA were evaluated by selective averaging of $R-R$ interval changes from multiple respiratory cycles over the respiratory phase. For the assessment of cardiorespiratory coordination, we analysed the phase-locking between heart rate, computed from R-R intervals of ECG, and respiratory rate, computed from intervals of inspiratory onsets of respiration, using Hilbert transform. A significant decrease in the magnitude of RSA and percentage and duration of phase-locking between heart rate and respiratory rate was observed during $U p$ as compared to Sup. Further, there was a significant increase in the phase of RSA during Up as compared to Sup. In conclusion, measures of RSA and interaction between cardiac and respiratory rhythms are affected by body posture in humans.
\end{abstract}

Keywords-heart, heart rate, phase locking, respiration, respiratory sinus arrhythmia

\section{INTRODUCTION}

The appearance of some relationship between two periodic oscillators in the form of locking of their phases or adjustment of rhythms can be termed as synchronization. Cardiorespiratory coordination is an aspect of the interaction between heart and respiratory rhythm which has been reported not only at rest [1,2,3] or during exercise [4], but also in subjects under the influence of anesthesia [5,6] and drugs [7,8,9]. Another well-known phenomenon of cardiorespiratory interaction is respiratory sinus arrhythmia (RSA) [10], which is a strong modulatory effect of respiration on heart rate [11].

Malliani et al. showed that changes in body posture can be considered as one of the simplest ways to produce an acute effect on parasympathetic activity [12]. Previous studies have reported the effect of postural changes on cardiovascular responses $[13,14]$. Although, a recent study by Gilad et al. presented the effect of changes in body posture on RSA [15], the association between body postures and cardiorespiratory coordination has never been established. In this study, we investigated the effect of body postures on the phase-locking between the cardiac and respiratory cycles in humans. We hypothesized that active standing reduces phase-locking compared to the supine position.

\section{METHODS}

\section{A. Subjects}

Eight subjects ( 4 males and 4 females) participated in this study. The study conformed to the principles outlined in the Declaration of Helsinki and was approved by the institution's human research ethics committee. Each subject provided written informed consent.

The protocol included 10 minutes of recording each in a supine and upright posture. ECG (leads I and II) and respiratory signals (from abdomen impedance belts) were sampled at $1 \mathrm{KHz}$ and acquired using a PowerLab A/D converter and ChartPro 6.0 software (ADInstruments, Sydney, Australia).

\section{B. Data Analysis}

1) $R-R$ Interval Analysis: Custom written computer software developed under MATLAB ${ }^{\circledR}$ was used to detect the R-peaks from the recorded ECG signal using parabolic fitting. The R-R time series obtained from the time-points of the R-peaks were visually scanned for artifacts.

2) Respiration Analysis: Respiratory signals consist of linear, nonlinear and non-stationary components, usually contaminated to some degree by noise. For our analysis, the respiratory signal was low-pass filtered at $0.5 \mathrm{~Hz}$. The inspiratory onsets, used to compute the breath-to-breath time series, were determined as the zero-crossings of the first derivative of the respiratory signal. 


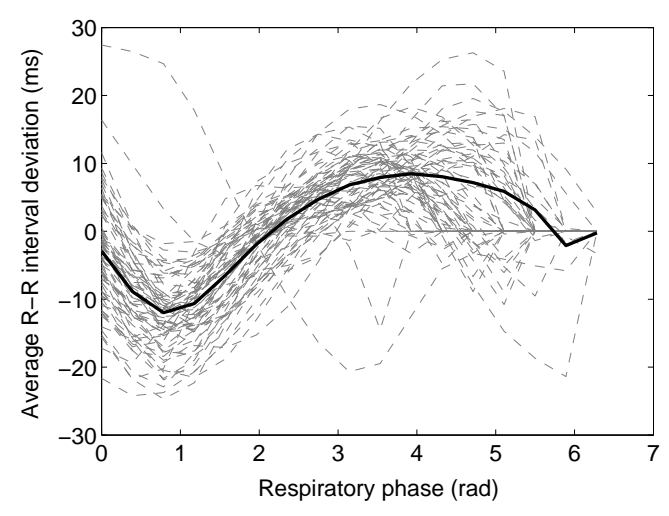

Fig. 1. Respiratory sinus arrhythmia (RSA) of a subject during supine position for $n$ respiratory cycles clustered together (dashed gray) and the overall RSA (solid line) obtained by averaging the clustered RSA.

3) RSA Pattern Analysis: For all $n$ respiratory cycles, the $\mathrm{R}-\mathrm{R}$ intervals between each two respiratory cycles were interpolated into 50 data points using cubic spline interpolation. The 50 data points for all $n$ respiratory cycles correspond to $2 \pi$ (Fig. 1). The overall RSA pattern was obtained by taking the average of all the RSA for $n$ respiratory cycles (Fig. 1).

4) Synchronization Analysis: We used Hilbert transform to calculate the phases of the respiratory signal, and determined relationship between the respiratory phases at different R-peak instants. Theoretically, if we denote the phase of heartbeat as $\Phi_{\mathrm{c}}$ and respiratory signal as $\Phi_{\mathrm{r}}$ and considering that the heart completes $m$ heartbeats in $n$ respiratory cycles, then the condition for phase locking can be given asheartbeats in $n$ respiratory cycles, then the condition for phase locking can be given as

$$
\left|m \Phi_{c}-n \Phi_{r}\right| \leq \text { const }
$$

In other words, if the phase difference between the two oscillators was within a certain threshold value and remained

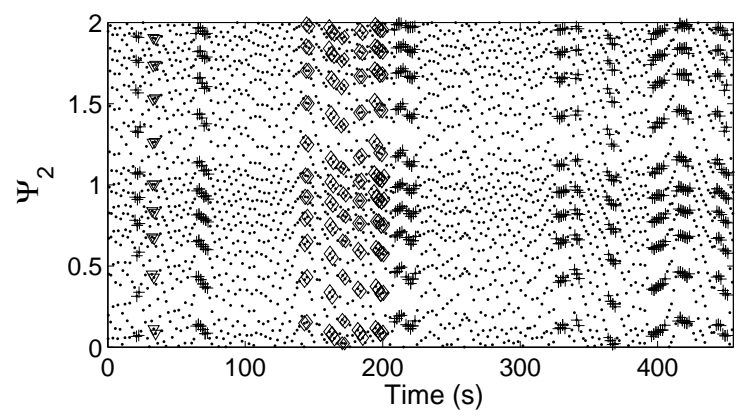

Fig. 2. Cardiorespiratory synchrogram plot showing time in seconds and the corresponding relative phases of heart beat normalized to 2 respiratory cycles. Dots indicate the normalized phases while delta, plus and diamond indicates 9:2, 10:2 and 11:2 phase-locked ratios respectively.

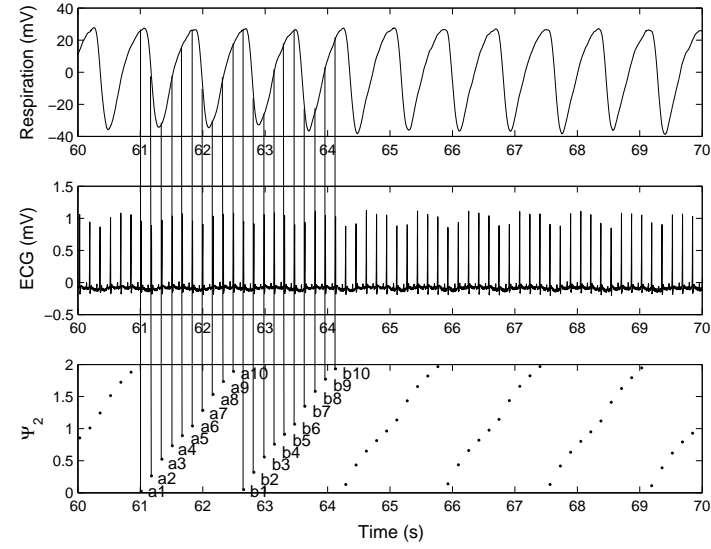

Fig. 3. Illustration of the generation of synchrogram from the respiratory and ECG signals. a1, a2, ., a10 and b1, b2, ..,b10 in the synchrogram plot represent the respiratory phases, based on the time points of R-peaks, for the first two and the following two respiratory cycles.

stable for $n$ respiratory cycles, the oscillators were considered synchronized. If $t_{k}$ is the time of the appearance of a $k^{\text {th }} \mathrm{R}$-peak, then by observing the phase of the respiration at the instants $t_{k}$, denoted by $\Phi_{\mathrm{r}}\left(t_{k}\right)$ and wrapping the respiratory phase into a $[0,2 \pi n]$ interval, we can generate cardiorespiratory synchrogram. This provides a visual tool to detect cardiorespiratory coordination (Fig. 2), by plotting $\Psi_{n}$ against $t_{k}$ which, in case of $m: n$ synchronization, results in $m$ horizontal lines. Here $\Psi_{n}$ is given by the equation

$$
\Psi_{n}=\frac{1}{2 \pi}\left(\Phi_{r}\left(t_{k}\right) \bmod 2 \pi n\right)
$$

In order to determine the values of $m$ and $n$, we selected one value of $n$ at a time and looked for coordinations at different values of $m$. The study was carried for the following $m: n$ coordinations: $n=1: m=2, \ldots, 7$ and $n=2: m=5,7,9,11,13$. We used a threshold value of 0.025 for the phase difference as it was suggested by Cysarz et al. [16].

We presented an illustration of the synchrogram plot in figure 3 . The phases of the respiratory signal, corresponding to the time points of the R-peaks, were plotted as normalized phases between 0 and 2. Subsequently, the phases for every two respiratory cycles formed a relatively vertical line: $\mathrm{a} 1, \mathrm{a} 2, \ldots, \mathrm{a} 10 ; \mathrm{b} 1, \mathrm{~b} 2, \ldots, \mathrm{b} 10$; and so on. We then determined the differences between each point of one line to each corresponding point of the next line: a1-b1, a2-b2,..,a10b10. If the differences between all the corresponding points were below the threshold value of 0.025 , the respective Rpeaks were considered as coordinated.

5) Statistical Analysis: We selected only artifact-free recording segments ( 3 to 4 segments for each subject) for the supine and upright postures to generate the results. Since the duration of each segment may vary, we calculated percentage of cardiorespiratory coordination by adding up 
TABLE 1

MEAN VALUES ( \pm STANDARD DEVIATION) OF THE R-R INTERVAL AND RESPIRATORY INTERVAL DURING SUPINE AND UPRIGHT BODY POSTURES

\begin{tabular}{|l|c|c|c|c|}
\hline \multirow{2}{*}{ Subjects } & \multicolumn{2}{|c|}{ R-R Interval (s) } & \multicolumn{2}{c|}{$\begin{array}{c}\text { Respiratory Interval } \\
\text { (s) }\end{array}$} \\
\cline { 2 - 5 } & Supine & Upright & Supine & Upright \\
\hline Subject 1 & $0.87 \pm 0.10$ & $0.61 \pm 0.05^{* * *}$ & $4.11 \pm 0.8$ & $4.61 \pm 1.0$ \\
\hline Subject 2 & $0.90 \pm 0.11$ & $0.72 \pm 0.08^{* * *}$ & $3.92 \pm 0.6$ & $4.13 \pm 0.9$ \\
\hline Subject 3 & $0.78 \pm 0.08$ & $0.65 \pm 0.04^{* * *}$ & $3.41 \pm 0.7$ & $3.23 \pm 0.8$ \\
\hline Subject 4 & $0.89 \pm 0.10$ & $0.64 \pm 0.05^{* * *}$ & $3.71 \pm 0.8$ & $4.21 \pm 1.1$ \\
\hline Subject 5 & $0.95 \pm 0.13$ & $0.76 \pm 0.10^{* * *}$ & $4.41 \pm 0.9$ & $4.70 \pm 1.2$ \\
\hline Subject 6 & $0.96 \pm 0.11$ & $0.68 \pm 0.09^{* * *}$ & $4.31 \pm 0.6$ & $4.54 \pm 0.8$ \\
\hline Subject 7 & $0.79 \pm 0.10$ & $0.60 \pm 0.04^{* * *}$ & $4.87 \pm 1.0$ & $5.01 \pm 1.3$ \\
\hline Subject 8 & $0.85 \pm 0.09$ & $0.67 \pm 0.05^{* * *}$ & $3.66 \pm 0.7$ & $4.41 \pm 0.9$ \\
\hline
\end{tabular}

Asterisks indicate differences with supine body posture.

$* * *$ represents $\mathrm{p}<0.0001$

the time for each coordinated epoch and then divided it by the total duration of the segments. Subsequently, we determined the percentage of the coordination during upright (Up) and supine (Sup) postures. We also recorded the phaselocking ratio and average duration of each coordinated epoch.

Statistical analysis was performed with GraphPad Prism ${ }^{\circledR}$ version 5.0 software (GraphPad, San Diego, CA, USA). Student's t-test was used to compare R-R intervals, respiratory intervals, RSA pattern and cardiorespiratory coordination between Up and Sup epochs. Data were expressed as mean $\pm \mathrm{SD}$. A value of $p<0.05$ was considered statistically significant.

\section{RESULTS}

\section{A. Effect of Body Posture on $R-R$ and Respiratory Interval}

All the subjects showed a shortening in R-R interval during Up as compared to Sup $(0.67 \pm 0.05$ vs. $0.87 \pm 0.12 \mathrm{~s}$, $\mathrm{p}<0.0001$, respectively) (Table 1 ). However, the respiratory interval was not significantly affected by body postures (Up vs. Sup: $4.4 \pm 0.8$ vs. $4.1 \pm 1.1 \mathrm{~s}, \mathrm{p}>0.05$ ) (Table 1 ).

\section{B. Effect of Body Posture on RSA}

The magnitude of RSA maxima in Up was significantly lower than in Sup for all subjects $(0.06 \pm 0.02$ vs. $0.13 \pm 0.04 \mathrm{~s}$, $\mathrm{p}<0.05$, respectively) (Table 2). On the other hand, the phase of the RSA pattern maxima was significantly higher in Up as compared to Sup $(5.2 \pm 2.1$ vs. $3.1 \pm 2.1$ rad, $\mathrm{p}<0.05$, respectively) (Table 2). The increase in phase of RSA maxima in Up indicates that the average RSA cycle is shifted towards to the right, as compared to Sup.

\section{Effect of Body Posture on Percentage of Cardiorespiratory Coordination}

The total percentage of coordination between cardiac and respiratory signals was significantly lower during Up as compared to Sup $(3.7 \pm 1.2$ vs. $15.2 \pm 2.1 \%, p<0.0001$, respectively) (Fig. 4).

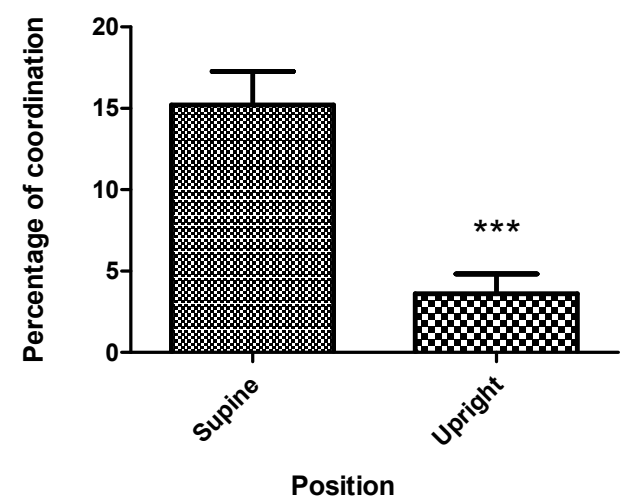

Fig. 4. Mean $\pm \mathrm{SD}$ of the percentage of coordination in all the subjects during supine and upright postures. Percentage of coordination was significantly higher during supine posture as compared to upright posture. ${ }^{* * *}$ represents $\mathrm{p}<0.0001$

\section{Effect of Body Posture on Duration of Cardiorespiratory Coordination}

A significant decrease in the average duration of cardiorespiratory coordination was observed during Up as compared to Sup $(4.6 \pm 2.1$ vs. $8.4 \pm 3.4 \quad \mathrm{~s}, \quad \mathrm{p}<0.05$, respectively) (Fig. 5).

\section{E. Effect of Body Posture on Phase-Locking Ratio}

The phase-locking ratio of $4: 1$ was the most frequently ratio observed during supine posture. Other phase-locking ratios for cardiorespiratory coordination were $2: 1,3: 1,5: 2$ and 7:2. However, in addition to a significant decrease in phase-locking between heart and respiratory cycles, 2:1 was the most frequently observed locking ratio during upright posture.

\section{Discussion}

This is the first study to investigate the interaction between cardiac and respiratory signals during supine (Sup) and upright (Up) body postures in humans.

TABLE 2

MEAN VALUES ( \pm STANDARD DEVIATION) OF THE MAXIMIM AMPLITUDE AND PHASE AT MAXIMUM AMPLITUDE OF RSA DURING SUPINE AND UPRIGHT BODY POSTURES

\begin{tabular}{|c|c|c|c|c|}
\hline \multirow{2}{*}{ Subjects } & \multicolumn{2}{|c|}{ Amplitude (s) } & \multicolumn{2}{c|}{ Phase (rad) } \\
\cline { 2 - 5 } & Supine & Upright & Supine & Upright \\
\hline Subject 1 & $0.11 \pm 0.04$ & $0.05 \pm 0.02^{*}$ & $2.65 \pm 1.8$ & $4.32 \pm 1.9^{*}$ \\
\hline Subject 2 & $0.14 \pm 0.04$ & $0.08 \pm 0.03^{*}$ & $3.42 \pm 1.7$ & $5.58 \pm 2.2^{*}$ \\
\hline Subject 3 & $0.15 \pm 0.05$ & $0.08 \pm 0.04^{*}$ & $2.84 \pm 1.5$ & $5.42 \pm 2.1^{*}$ \\
\hline Subject 4 & $0.12 \pm 0.03$ & $0.04 \pm 0.02^{*}$ & $2.98 \pm 2.1$ & $4.77 \pm 1.6^{*}$ \\
\hline Subject 5 & $0.15 \pm 0.04$ & $0.06 \pm 0.03^{*}$ & $3.23 \pm 1.7$ & $5.56 \pm 1.8^{*}$ \\
\hline Subject 6 & $0.13 \pm 0.05$ & $0.05 \pm 0.02^{*}$ & $2.71 \pm 1.8$ & $4.63 \pm 1.9^{*}$ \\
\hline Subject 7 & $0.12 \pm 0.04$ & $0.06 \pm 0.03^{*}$ & $3.12 \pm 1.5$ & $5.31 \pm 1.4^{*}$ \\
\hline Subject 8 & $0.13 \pm 0.04$ & $0.05 \pm 0.02^{*}$ & $3.67 \pm 1.6$ & $5.62 \pm 1.5^{*}$ \\
\hline
\end{tabular}

Asterisks indicate differences with supine body posture.

* represents $\mathrm{p}<0.05$. 


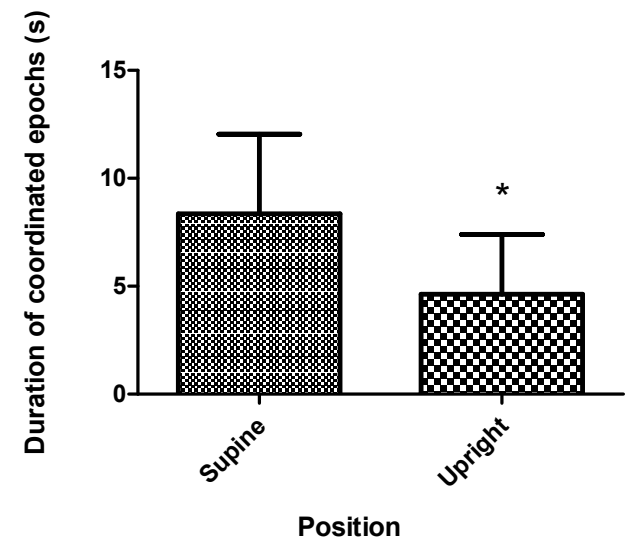

Fig. 5. Mean \pm SD of the average duration of coordinated epochs in all the subjects during supine and upright postures. The average duration of coordinated epochs was significantly higher during supine posture as compared to upright posture. ${ }^{*}$ represents $\mathrm{p}<0.05$

Our results show that the amount of interaction between the cardiac and respiratory oscillators is decreased during Up as compared to Sup. It is also evident that Up, as compared to Sup, causes a shortening in the R-R intervals with no significant change in respiratory intervals, which is consistent with the findings by Gilad et al. [15]. Similarly, our study shows a decrease and increase in the amplitude and phase of RSA maxima in Up, as reported earlier [15].

It is well known that a postural change in human body from upright to supine causes an increase in vagus nerve activity and a decrease in sympathetic nerve activity $[17,18$, 19]. The decrease in sympathetic nervous activity together with the increase in vagal activity in Sup is the most likely cause of an increase in R-R interval. The decrease in amplitude of RSA in Up is in agreement with the literature $[15,20]$, indicating less influence of respiration on heart rate in upright standing posture.

The phase-coupling between cardiac and respiratory rhythms has long been recognized [1,2,3]. Although the physiological significance of cardiorespiratory coordination is yet to be established, the relationship between heart rate and respiration has been suggested as a useful tool for assessing the autonomic nervous system functioning [21], diagnosis of sepsis [22] and obstructive sleep apnea [23]. However, for the accurate determination of the coordination process, the ratio of heart rate to respiratory rate should also be considered [24]. In this study, a significant decrease in cardiorespiratory coordination was observed in Up as compared to Sup. Up also caused a change in the most frequent phase-locking ratio from $4: 1$ to $2: 1$. It is interesting to observe that although the heart rate increases in Up, there is a decrease in the overall phase-locking ratio. According to a recent study, the autonomic nervous system has an influence on cardiorespiratory coordination [9]. This would suggest that a disturbance in autonomic nervous activity caused by the postural changes is the dominant cause of the increase or decrease in cardiorespiratory coordination.

\section{CONCLUSION}

Upright standing posture in humans compared to supine posture is associated with a reduction of phase-locking between cardiac and respiratory cycles.

\section{REFERENCES}

[1] C. Schafer, M. G. Rosenblum, J. Kurths and H. H. Abel, "Heartbeat synchronized with ventilation," Nature, vol. 392, pp. 239-240, 1998.

[2] C. Schafer, M. G. Rosenblum, H. H. Abel and J. Kurths, "Synchronization in the human cardiorespiratory system," Phys Rev E Stat Phys Plasmas Fluids Relat Interdiscip Topics, vol. 60, pp. 857$870,1999$.

[3] M. B. Lotric and A. Stefanovska, "Synchronization and modulation in the human cardiorespiratory system," Physica A, vol. 283, pp. 451461,2000 .

[4] D. Kenwright, A. Bahraminasab, A. Stefanovska and P. V. E. McClintock, "The effect of low-frequency oscillations on cardiorespiratory synchronization," Eur Phys J B Condensed Matter and Complex Systems, vol. 65, pp. 425-433, 2008.

[5] D. C. Galletly and P. D. Larsen, "Cardioventilatory coupling during anesthesia,” Br J Anaesth, vol. 79, pp. 35-40, 1997.

[6] M. M. Kabir, M. I. Beig, E. Nalivaiko, D. Abbott, and M. Baumert, "Isoflurane increases cardiorespiratory coordination in rats," in Biomedical Applications of Micro- and Nanoengineering IV and Complex Systems, Melbourne, Australia, 2008, pp. 72700Y-7.

[7] A. Stefanovska, H. Haken, P. V. E. McClintock, M. Hozic, F. Bajrovic and S. Ribaric, "Reversible transitions between synchronization states of the cardiorespiratory system," Phys Rev Lett, vol. 85, pp. 4831-4834, 2000.

[8] G. Marano, M. Grigioni, F. Tiburzi, A. Vergari and F. Zanghi, "Effects of isoflurane on cardiovascular system and sympathovagal balance in New Zealand white rabbits," J Cardiovasc Pharmacol, vol. 28, pp. 513-518, 1996.

[9] M. M. Kabir, M. I. Beig, E. Nalivaiko, D. Abbott, and M. Baumert, "Cardiorespiratory Coordination in Rats is Influenced by Autonomic Blockade," in 13th International Conference on Biomedical Engineering, 2009, pp. 456-459.

[10] G. G. Berntson, J. T. Cacioppo and K. S. Quigley, "Respiratory sinus arrhythmia: autonomic origins, physiological mechanisms, and psychophysiological implications," Psychophysiology, vol. 30, pp. 183-196, 1993.

[11] L. Bernardi, C. Porta, A. Gabutti, L. Spicuzza and P. Sleight, "Modulatory effects of respiration," Auton Neurosci, vol. 90, pp. 4756, 2001.

[12] A. Malliani and N. Montano, "Heart rate variability as a clinical tool," Ital Heart J, vol. 3, pp. 439-445, 2002.

[13] T. Takahashi, A. Okada, T. Saitoh, J. Hayano and Y. Miyamoto, "Difference in human cardiovascular response between upright and supine recovery from upright cycle exercise," Eur J Appl Physiol, vol. 81, pp. 233-239, 2000.

[14] T. Takahashi, J. Hayano, A. Okada, T. Saitoh and A. Kamiya, "Effects of the muscle pump and body posture on cardiovascular responses during recovery from cycle exercise," Eur J Appl Physiol, vol. 94, pp. 576-583, 2005.

[15] O. Gilad, C. A. Swenne, L. R. Davrath and S. Akselrod, "Phaseaveraged characterization of respiratory sinus arrhythmia pattern," Am J Physiol Heart Circ Physiol, vol. 288, pp. H504-H510, 2005.

[16] D. Cysarz, H. Bettermann, S. Lange, D. Geue and P. V. Leeuwen, "A quantitative comparison of different methods to detect cardiorespiratory coordination during night-time sleep," Biomed Eng Online, vol. 3, 2004. 
[17] B. F. Robinson, S. E. Epstein, G. D. Beiser and E. Braunwald, "Control of heart rate by the autonomic nervous system: Studies in man on the interrelation between baroreceptors mechanisms and exercise," Circ Res, vol. 19, pp. 400-411, 1966.

[18] L. B. Rowell, "Reflex control during orthostasis," in Human cardiovascular control, L. B. Rowell, Ed. Oxford University Press, New York, pp. 37-80, 1993.

[19] M. Buchheit, H. Al Haddad, P. B. Laursen and S. Ahmaidi, "Effect of body posture on postexercise parasympathetic reactivation in men," Exp Physiol, vol. 94, pp. 795-804, 2009.

[20] J. P. Saul, R. D. Berger, P. Albrecht, S. P. Stein, M. H. Chen and R. J. Cohen, "Transfer function analysis of the circulation: unique insights into cardiovascular regulation," Am J Physiol Heart Circ Physiol, vol. 261, pp. H1231-H1245, 1991.

[21] D. Hoyer, U. Leder, H. Hoyer, B. Pompe, M. Sommer and U. Zwiener, "Mutual information and phase dependencies: measures of reduced nonlinear cardiorespiratory interactions after myocardial infarction," Med Eng Phys, vol. 24, pp. 33-43, 2002.

[22] R. Loforte, G. Carrault, L. Mainardi and A. Beuche, "Heart rate and respiration relationships as a diagnostic tool for late onset sepsis in sick preterm infants," Computers in Cardiology, vol. 33, pp. 737-740, 2006.

[23] M. M. Kabir, H. Dimitri, P. Sanders, R. Antic, E. Nalivaiko, D. Abbott, and M. Baumert, "Cardiorespiratory Phase-Coupling Is Reduced in Patients with Obstructive Sleep Apnea," PLOS ONE, vol. 5, p. e10602, 2010.

[24] Y. C. Tzeng, P. D. Larsen, D. C. Galletly, "Mechanism of cardioventilatory coupling: insights from cardiac pacing, vagotomy, and sinoaortic denervation in the anesthetized rat." Am J Physiol Heart Circ Physiol, vol. 292, pp. H1967-1977, 2007. 